\title{
Comparison of fluctuations properties measured by Langmuir and by ball-pen probes in the ISTTOK boundary plasma
}

\author{
C. Silva ${ }^{1}$, J. Adamek ${ }^{2}$, H. Fernandes ${ }^{1}$, P. Duarte ${ }^{1}$ \\ ${ }^{1}$ Associação Euratom/IST, Instituto de Plasmas e Fusão Nuclear, Instituto Superior Técnico, \\ Universidade Técnica de Lisboa, Portugal \\ ${ }^{2}$ Institute of Plasma Physics AS CR, Association EURATOM/IPP.CR, Praha 8, Czech Republic
}

\section{Introduction}

Langmuir probes (LPs) are extensively used in the boundary of fusion devices providing most of the data on the local electron temperature, potential and plasma density. They allow the simultaneous determination of several plasma parameters with high spatial and temporal resolution justifying its continued use, particularly for turbulent transport studies. Density and plasma potential fluctuations are typically evaluated from the ion saturation current and floating potential, respectively, neglecting the influence of the electron temperature fluctuations. This approach is often questioned as the properties of the floating and plasma potential fluctuations appear to be significantly different [1-5]. Recently, ball-pen probes (BPPs) were found to be well-suited for routine measurements of the plasma potential in the edge plasma of fusion devices $[3,5]$.

This work aims at comparing the fluctuations properties measured by Langmuir and ball-pen probes, including the cross-field turbulent particle flux that should be more accurately estimated with BPPs. Different probe systems combining LP and BPPs has been developed on ISTTOK to simultaneously measure the floating and plasma potential, permitting also the determination of the turbulent particle flux using both types of probes.

\section{Experimental set-up}

Three probe systems were used in this work that are illustrated in figure 1: (a) a single ballpen probe with a movable conductor of $4 \mathrm{~mm}$ in diameter covered by a boron nitride tube with an outer diameter of $7 \mathrm{~mm}$ (figure 1a); (b) a 3-pin probe head consisting of one cylindrical pin (with a diameter of $0.75 \mathrm{~mm}$ and an exposed length of $2.5 \mathrm{~mm}$ ) and two ballpen type probes (poloidally separated by $7 \mathrm{~mm}$ ), each with a conductor of $3 \mathrm{~mm}$ in diameter recessed by $1 \mathrm{~mm}$ with respected to the probe surface (figure 1b); (c) a poloidal array of 9 pins separated by $2 \mathrm{~mm}$, with the central pin measuring the ion saturation current, $\mathrm{I}_{\text {sat }}$, and the remaining measuring the floating potential, $V_{\mathrm{f}}$. Probes $b$ ) and c) permit the determination of the turbulent particle flux that is usually estimated using $\Gamma_{E \times B}=\left\langle\tilde{n} \tilde{E}_{\theta}\right\rangle / B$, where $\tilde{n}$ and $\tilde{E}_{\theta}$ are density and poloidal electric field fluctuations, respectively. Plasma parameters have been measured in the scrape-off layer (SOL) and in the edge plasma (up to $2 \mathrm{~cm}$ inside the last closed flux surface, LCFS) and signals simultaneously sampled at $2 \mathrm{MHz}$. 


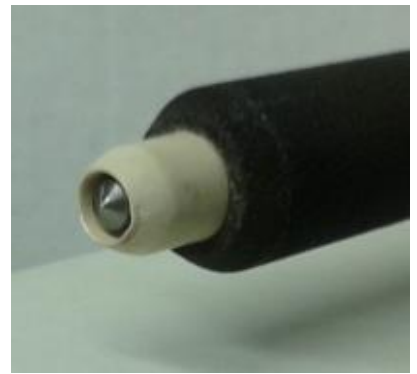

a)

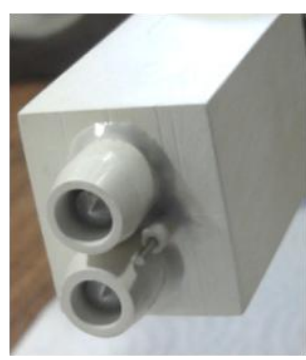

b)

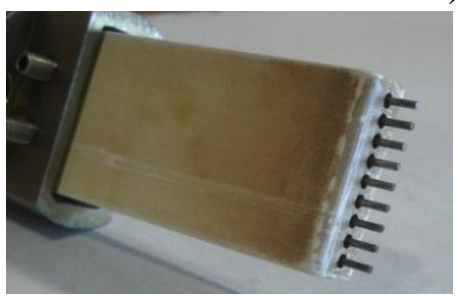

Figure 1: Probe systems used on ISTTOK

\section{Experimental results}

\subsection{BPP I -V Characteristic}

Ideally, the ratio of the ion to electron currents of the BPP should be approximately one so that the probe floats at the plasma potential. Figure 2 shows the BPP I-V characteristic for two discharges. The ratio of the probe currents is experimentally observed to be in the range 1 -2.5 , although a ratio of one is rarely observed. This means that in general the BPP will not float at the plasma potential but up to $\sim T_{e} / e$ below that value. For a $\mathrm{H}^{+}$plasma with $\mathrm{T}_{\mathrm{e}} \approx \mathrm{T}_{\mathrm{i}}$ LPs should float at $\sim 3 \mathrm{~T}_{\mathrm{e}} / e$ below the plasma potential and consequently it is expected that the potential measured by the BPP is typically $2 \mathrm{~T}_{\mathrm{e}} / e$ above the floating potential measured by

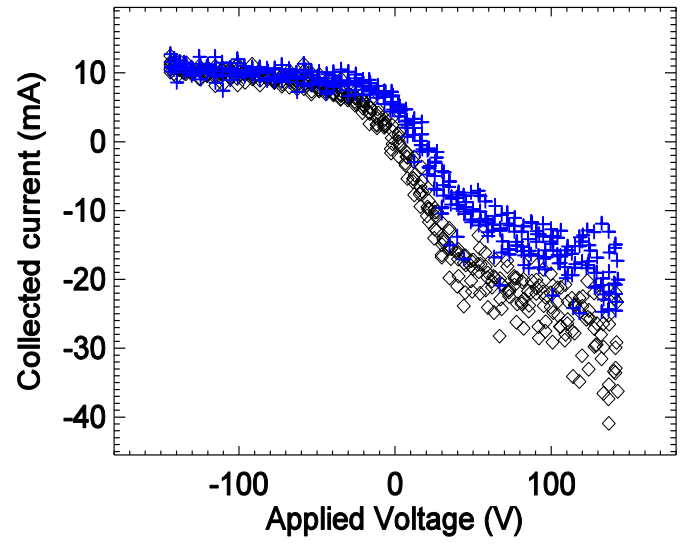

Figure 2: BPP I-V characteristic for two discharges with probe located $1.5 \mathrm{~cm}$ inside the LCFS. a LP.

\subsection{Comparison of the floating potential measured by Langmuir and ball-pen probes}

The floating potential estimated simultaneously by $\mathrm{LP}\left(\mathrm{V}_{\mathrm{f}}^{\mathrm{LP}}\right)$ and BPP $\left(\mathrm{V}_{\mathrm{f}}^{\mathrm{BPP}}\right)$ using the 3-pin probe have been compared. As illustrated in figure 3a, that shows the temporal evolution of the floating potential fluctuations measured by both type of probes, the amplitude of the fluctuations in $\mathrm{V}_{\mathrm{f}}^{\mathrm{LP}}$ is clearly larger than that in $\mathrm{V}_{\mathrm{f}}^{\mathrm{BPP}}$, confirming that BPP measurements are less affected by the temperature fluctuations. It should be noted, however, that the temporal evolution of the two signals is similar and that fluctuations are roughly in phase as confirmed by cross-correlation between them. Results suggest that temperature and plasma potential fluctuations are in phase opposition so that $V_{f}=V_{p}-3 T_{e}$ is in phase with $V_{p}$.

Figure $3 \mathrm{~b}$ shows the frequency spectra of the floating potential measured by both type of probes indicating that $\mathrm{V}_{\mathrm{f}}^{\mathrm{LP}}$ fluctuations are larger across most of the spectra but particularly at high frequencies ( $\mathrm{f}>100 \mathrm{kHz}$ ). Langmuir probe potential signals have therefore a higher average frequency and consequently a lower auto-correlation time. 
Taking as example the data shown in figure 3 , we have for $V_{\mathrm{f}}{ }^{\mathrm{BPP}}$ a standard deviation of $4 \mathrm{~V}$, an average frequency of $90 \mathrm{kHz}$ and an autocorrelation time of $6 \mu$ s while for $\mathrm{V}_{\mathrm{f}}^{\mathrm{LP}}$ a standard deviation of $7.2 \mathrm{~V}$ an average frequency of 101 $\mathrm{kHz}$ and an autocorrelation time of 5.5 $\mu s$. The higher average frequency of the $\mathrm{V}_{\mathrm{f}}^{\mathrm{LP}}$ signals is not a consequence of the smaller diameter of the LP pin as similar results are obtained with the single BPP operated as a LP (conductor exposed to the plasma).

The poloidal electric field has also been estimated using both types of probes, although not simultaneously. The amplitude of the fluctuations is larger when measured with LP although in this case a larger difference is observed at low frequencies $(<40 \mathrm{kHz})$.
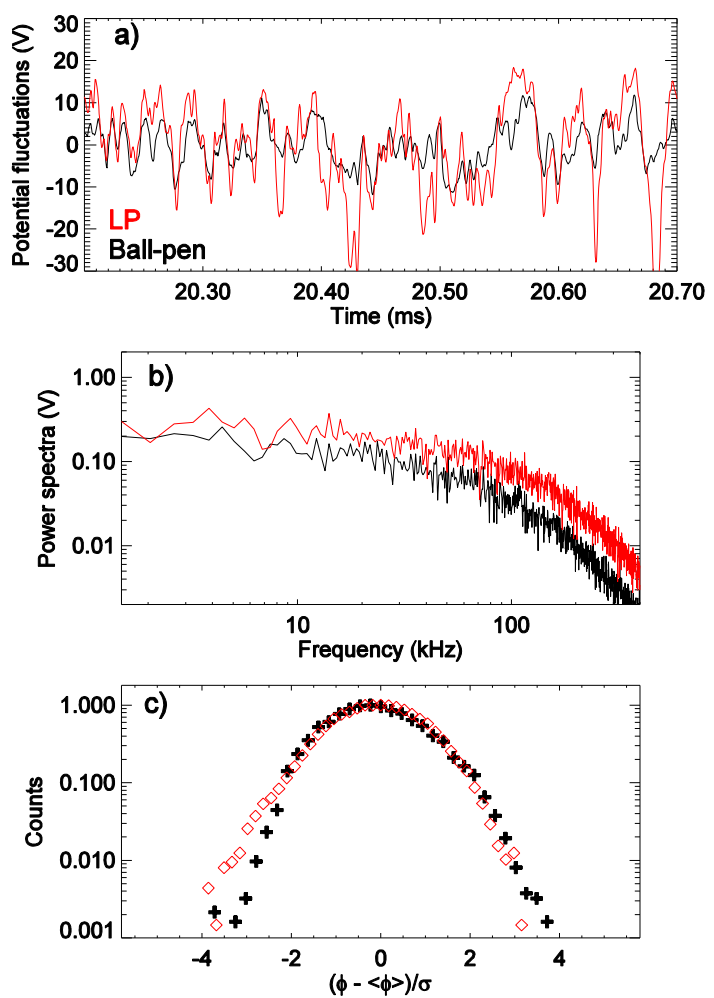

Figure 3: Temporal evolution of the potential fluctuations measure by LP and BPP (a) together with the respective frequency spectra (b) and PDFs (c)

To conclude the comparison between $\mathrm{V}_{\mathrm{f}}$ signals measured by LP and BPP the probability distribution function has been estimated (Figure 3c). The PDFs normalized to the standard deviation are very similar, being a modest difference in skeweness observed: $S\left(V_{f}{ }^{B P P}\right)=0.2$, $\mathrm{S}\left(\mathrm{V}_{\mathrm{f}}^{\mathrm{LP}}\right)=-0.2$. In summary, the main different in the $\mathrm{V}_{\mathrm{f}}$ fluctuations properties measured by LP and BPP is in the standard deviation that is up to two times larger when measured with LPs.

\subsection{Radial Profiles}

The radial profiles of different plasma quantities measured by the two types of probes have been compared with results illustrated in figure 4. As expected, the floating potential measured by the BPP is larger than that measured by the LP. This potential difference can be used to estimate the electron temperature using the relation $T_{e} \approx\left(V_{f}^{B P P}-V_{f}^{L P}\right) / 2$. Also shown is the temperature estimated from the standard swept LP that is 5 to $10 \mathrm{eV}$ above the value measured with the BPP. It should be noted that the $T_{e}$ obtained in the SOL by the BPP (around $1 \mathrm{eV}$ ) seems to be unrealistically low, although no other diagnostic exists to validate the measurements. This is an intriguing result as the ion to electron saturation current ratio observed in the SOL is low (about 2) and therefore the BPP measurements appear to be valid. The amplitude of the potential and poloidal electric field fluctuations are in general smaller 
when measured with the BPP apart from the SOL where a reasonable agreement is observed. A similar conclusion is observed for the cross-field turbulent flux, which is up to 2.5 times larger when estimated with standard LPs in the region just inside the LCFS. This larger turbulent flux is mainly observed at low frequencies $(<50 \mathrm{kHz})$ resulting mostly from the larger amplitude of the poloidal electric field in this
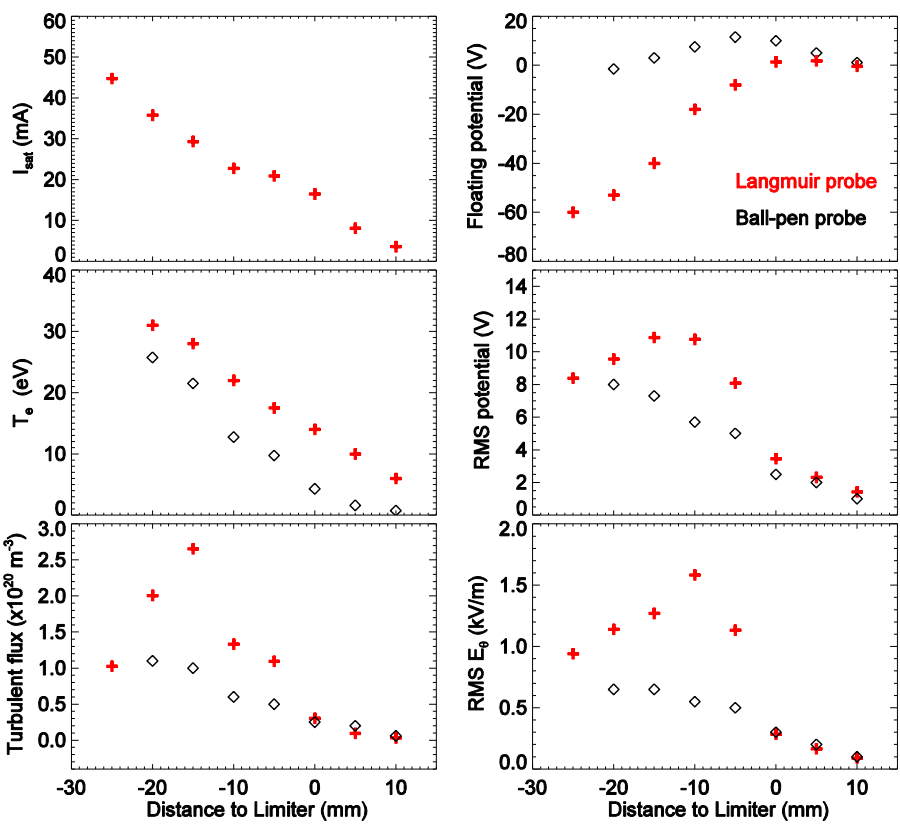

Figure 4: Radial profile of the main parameters derived from LP and BPP.

frequency range.

\section{Conclusions}

ISTTOK results indicate that the amplitude of the floating potential fluctuations measured by Langmuir probes is larger than that measured by ball-pen probes, confirming that the latter measurements are less affected by the temperature fluctuations. Furthermore, the turbulent particle flux measured using BPPs was also found to be up to 2.5 times larger in the region just inside the LCFS when measured with LPs. However, properties of the potential fluctuations such as the PDF and the spectra are not significantly different when measured by LP and BPP. Further work is required for instance to clarify the differences in the electron temperature obtained from the two methods, particularly in SOL, and for a wider validation of the Langmuir and Ball-pen probe measurements.

\section{Acknowledgements}

This work was supported by EURATOM and carried out within the framework of the European Fusion Development Agreement. IST activities also received financial support from "Fundação para a Ciência e Tecnologia" through project Pest-OE/SADG/LA0010/2011. Additional support has been granted from IAEA technical contract CRP F1.30.14 on "Utilization of the Network of Small Magnetic Confinement Fusion Devices for Mainstream Fusion Research". The views and opinions expressed herein do not necessarily reflect those of the European Commission.

\section{References}

[1] B. Nold et al., New Journal of Physics 14 (2012) 063022

[2] F. Gennrich and K. Kendl, Plasma Phys. Control. Fusion 54 (2012) 015012

[3] J. Adamek et al., Contributions to Plasma Physics 50 (2010) 854

[4] J. Adamek et al., Czechoslovak Journal of Physics, 52 (2002) 115

[5] J. Adamek et al., $32^{\text {nd }}$ EPS Conf. Plasma Phys, Spain, (2005), P5.085 